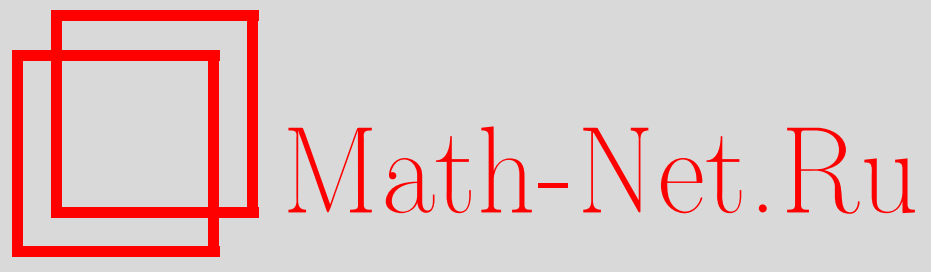

Л. Б. Шапиро, Об универсальных топологических алгебрах типа Валдивиа, УМН, 2008, том 63, выпуск 3, 167168

DOI: https://doi.org/10.4213/rm9177

Использование Общероссийского математического портала Math-Net.Ru подразумевает, что вы прочитали и согласны с пользовательским соглашением http://www . mathnet.ru/rus/agreement

Параметры загрузки:

IP : 3.89 .185 .249

26 апреля 2023 г., 15:47:53

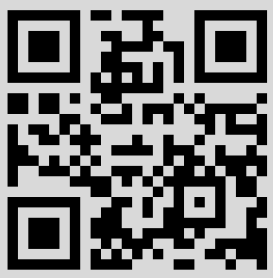




\section{Об универсальных топологических алгебрах типа Валдивиа}

\section{Л. Б. Шапиро}

В заметке рассматривается вопрос о том, когда подалгебра прямого произведения универсальных топологических алгебр имеет структуру произведения "более простых" сомножителей. В первой части приводятся необходимые определения и основные результаты для конкретных случаев. Во второй части описывается наиболее общий, с точки зрения автора, подход к поставленному выше вопросу.

1. Согласно теореме Чейза [1], для любой области главных идеалов (о.г.и.) $R$, наделенной дискретной топологией, замкнутый подмодуль $R^{\omega}$ изоморфен ${ }^{1}$ степени $R$. Ранее Нунке [2] установил аналогичный факт для $\mathbb{Z}$. Нашей целью является обобщение теоремы Нунке-Чейза на несчетный случай. Для этого, по аналогии с компактами Валдивиа [3], мы вводим

ОПРедЕЛЕНИЕ 1 . Пусть $\left\{\left(X_{i}, x_{i}^{*}\right): i \in I\right\}$ - семейство (несчетное) топологических пространств с отмеченными точками $x_{i}^{*} \in X_{i}$. Подпространство $Y \subset \prod\left\{X_{i}: i \in I\right\}$ имеет тип Валдивиа, если $\left\{y=\left\{y_{i}\right\} \in Y:\left|\left\{i: y_{i} \neq x_{i}^{*}\right\}\right| \leqslant \omega\right\}$ всюду плотно в $Y$, т. е. след, который $\Sigma$-произведение $\left\{X_{i}: i \in I\right\}$ (см., например, [3]) "оставляет" на $Y$, плотен в нем. Для алгебраических структур (колец, тел, алгебр) отмеченной точкой считается нейтральный элемент.

Следующие два результата являются основными.

Теорема 1. Пусть $R$ - область главных идеалов, наделенная дискретной топологией. Тогда замкнутый подмодуль (над $R$ ) типа Валдивиа несчетной степени $R^{\tau}$ изоморфен степени $R$.

Теорема 2. Пусть $K$ - полное недискретное нормированное ${ }^{2}$ тело. Тогда замкнутое линейное подпространство (над $K$ ) типа Валдивиа несчетной степени $K^{\tau}$ изоморфно степени $K$.

Доказательства приведенных теорем проходят по общей схеме и основаны на следующих четырех леммах.

ЛЕмма 1. Пусть $E, F$ - конечнопорожденные модули над о.г.и. (п.н.н. телом) u $f: E \rightarrow F$ - алгебраический гомоморфизм. Тогда $f$ - тривиальное расслоение со слоe⿻ $\operatorname{Ker} f$.

Доказательство леммы 1 для случая о.г.и. можно найти в [4] (гл. XV, § 2, лемма 1), а для случая п.н.н. тела - в [5] (гл. I, § 2, п. 3, предложение 3). Из леммы 1 с помощью спектральной аппроксимации получаем

СледСтвие 1. Замкнутый подмодуль счетной степени о.г.и. (п.н.н. тела) Е изоморфен степени Е.

Лемма 2. Пусть $Е$ - о.г.и. (п.н.н. тело). Тогда $E^{\omega}$ удовлетворяет следующим свойствам:

а) любой подмодуль $E^{\omega}$ топологически счетнопорожден, т. е. существует счетное подмножество, алгебраически порождающее всюду плотную часть;

б) любал возрастающая по типу $\omega_{1}$ цепочка замкнутых подмодулей стабилизиpуется.

\footnotetext{
${ }^{1}$ Под изоморфизмом тополого-алгебраических объектов понимается алгебраический изоморфизм, который одновременно является гомеоморфизмом.

2 Далее используется аббревиатура п.н.н.
} 
Как обычно, для отображения $f: X \rightarrow X$ подмножество $Y \subset X$ называется $f$-инвариантным, если $f(Y) \subset Y$. Для семейства пространств с отмеченными точками $\left\{\left(X_{i}, x_{i}^{*}\right): i \in I\right\}$ и подмножества $J \subset I$ естественная проекция-ретракция $\operatorname{pr}_{J}: \sum\left\{X_{i}:\right.$ $i \in I\} \rightarrow \sum\left\{X_{i}: i \in I\right\}$ определяется так: $i$-я координата точки $\operatorname{pr}_{J}(x)$ совпадает с $x_{i}$, если $i \in J$, и равна $x_{i}^{*}$, если $i \notin J$.

Следующая лемма возникла в результате знакомства с работами [6], [7].

Лемма 3. Пусть $E$ - о.г.и. (п.н.н. тело) и $M$ - замкнутый подмодуль $\Sigma$-произведения несчетного числа экземпляров $E$, заиндексированных множеством I. Тогда для любого счетного подмножества $J \subset I$ существует счетное подмножество $J^{\prime} \subset J$ такое, что $M$ является $\operatorname{pr}_{J^{\prime}}$-инвариантным.

ОпредЕлЕниЕ 2. Следуя [8], группоид с нейтральным элементом, в котором каждый элемент обратим, будем называть саморефлексивным в слабом смысле.

Важную роль в доказательствах играет простая

Лемма 4. Пусть $G_{1}, G_{2}, H$ - группоиды, саморефлексивные в слабом смысле, причем $H \leqslant G_{1} \times G_{2}$ и $H$ является $\mathrm{pr}_{1}$-инвариантным подмножеством, где $\mathrm{pr}_{1}: G_{1} \times$ $G_{2} \rightarrow G_{1} \times G_{2}$ определено равенством $\operatorname{pr}_{1}\left(g_{1}, g_{2}\right)=\left(g_{1}, e_{2}\right)$. Тогда $H=H_{1} \times H_{2}$, где $H_{1} \leqslant G_{1} u H_{2} \leqslant G_{2}$.

2. Анализ доказательств теорем 1, 2 приводит к мысли о введении общих понятий в рамках теории универсальных топологических алгебр [9].

ОПредЕлЕниЕ 3. Универсальную алгебру назовем алгеброй Бэра, если она является укрупнением (см. [10; с. 84]) саморефлексивного в слабом смысле группоида.

ОпредЕлЕние 4. Универсальная топологическая алгебра называется $\omega_{1}$-топологически нётеровой, если любая ее подалгебра топологически счетнопорождена или, эквивалентно, любая возрастающая по типу $\omega_{1}$ цепочка замкнутых подалгебр стабилизируется.

Теорема 3. Пусть $\left\{\left(A_{i}, \Omega\right): i \in I\right\}$ - несчетное семейство топологических алгебр Бэра одной сигнатуры, каждое конечное подпроизведение $\omega_{1}$-топологически нётерово и В - замкнутал подалгебра типа Валдивиа произведения (П\{ $\left.\left.A_{i}: i \in I\right\}, \Omega\right)$. Тогда существуют $\left\{I_{t}: t \in T\right\}$-разбиение $I$ на счетные попарно дизбюнктные множества и замкнутые подалгебры $B_{t} \leqslant \prod\left\{A_{i}: i \in I_{t}\right\}$ такие, что $B=\prod\left\{B_{t}: t \in T\right\}$.

СледСтвие 2. Пусть $\left\{G_{i}: i \in I\right\}$ - несчетное семейство топологических групn со второй аксиомой счетности и $H$ - замкнутая подгруппа типа Валдивиа произведения $\prod\left\{G_{i}: i \in I\right\}$. Тогда $H$ изоморфна произведению групп со второй аксиомой счетности.

Автор выражает благодарность В. Марчишевскому за полезную консультацию и А. П. Комбарову за дружескую поддержку, без которой эта работа вряд ли была бы выполнена.

\section{Список литературы}

[1] S. U. Chase, Illinois J. Math., 7 (1963), 593-608. [2] R. J. Nunke, Proc. Amer. Math. Soc., 13:1 (1962), 66-71. [3] O. Kalenda, Collect. Math., 51:1 (2000), 59-81. [4] С. Ленг, Алгебра, Мир, М., 1968. [5] Н. Бурбаки, Топологические векторные пространства, ИЛ, М., 1959. [6] А. П. Комбаров, В. И. Малыхин, Докл. АН СССР, 213 (1973), 774-776. [7] С. П. Гулько, Докл. АН СССР, 237:3 (1977), 505-508. [8] R. Baer, Amer. J. Math., 71:3 (1949), 706-742. [9] А. И. Мальцев, Матем. сб., 35:1 (1954), 3-20. [10] P. М. Cohn, Universal algebra, Harper \& Row, New York-London, 1965.

Л. Б. Шапиро (L. B. Shapiro)

Академия труда и социальных отношений

E-mail: 1shapiro@mccme.ru
Представлено С. М. Гусейн-Заде Принято редколлегией 24.01.2008 